\title{
Fraturas osteoporóticas da coluna vertebral
}

\author{
João Welberthon Matos Queiroz', Paula Camila Alves de \\ Assis Pereira ${ }^{1}$, Eberval Gadelha Figueiredo ${ }^{2}$
}

Universidade Federal de Campina Grande (UFCG), Cajazeiras, PB, Brasil. Divisão de Neurocirurgia do Hospital das Clínicas da Faculdade de Medicina da Universidade de São Paulo (HCFMUSP), São Paulo, SP, Brasil.

\section{RESUMO}

As fraturas vertebrais são comuns em idosos e em mulheres no período pós-menopausa. Tais fraturas podem ter como etiologia principal a osteoporose ou ser decorrentes de trauma, infecções ou neoplasia. A osteoporose é caracterizada por diminuição da massa e modificação da microarquitetura óssea, sendo a manifestação mais comum a fratura patológica. Apresenta como manifestação clínica dor, diminuição da altura e desalinhamento da coluna vertebral, sintomas neurológicos, bem como alteração na autoestima e problemas sociais. O diagnóstico de osteoporose é dado por meio da realização de densitometria óssea, sendo esse o padrão-ouro, podendo-se lançar mão de outros métodos de imagem em situações específicas. O tratamento da osteoporose inclui métodos para a prevenção de fraturas, tais como reposição de cálcio e vitamina $D$, uso de bifosfonados, paratormônio e reposição hormonal. Quando em vigência de fratura, a conduta preferida ainda é a abordagem não cirúrgica, sendo feita por meio de imobilização, uso de analgésicos, fisioterapia e reabilitação motora. Quando em vigência de déficit neurológico, deformidade severa ou ausência de resposta ao tratamento conservador, está indicada a abordagem cirúrgica.

\section{PALAVRAS-CHAVE}

Coluna vertebral, fraturas osteoporóticas, osteoporose.

\section{ABSTRACT}

\section{Osteoporotic fractures spine}

Vertebral fractures are common in the elderly and in women in the post menopausal period. Such fractures can have as cause osteoporosis or be due to trauma, infection or neoplasia. Osteoporosis is characterized by decreased bone mass and modified microarchitecture, the most common manifestation of a pathological fracture. Presenting clinical manifestation as pain, height loss and misalignment of the spine, neurological symptoms, and change in self-esteem and social problems. The diagnosis of osteoporosis is given by bone densitometry, this being the gold standard, and we can make use of other imaging methods in specific situations. Treatment of osteoporosis includes methods for the prevention of fractures, such as treatment with calcium and vitamin $D$, use of bisphosphonates, parathyroid hormone and hormone replacement. When in the presence of fracture, the preferred approach is still nonsurgical approach being taken by immobilization, analgesics, physical therapy and motor rehabilitation. When in the presence of neurologic deficit, severe deformity or lack of response to conservative treatment require surgical approach.

\section{KEYWORDS}

Spine, osteoporotic fractures, osteoporosis.

1 Graduando em Medicina pela Universidade Federal de Campina Grande (UFCG), Cajazeiras, PB, Brasil.

2 Divisão de Neurocirurgia do Hospital das Clínicas da Faculdade de Medicina da Universidade de São Paulo (HCFMUSP), São Paulo, SP, Brasil. 


\section{Introdução}

As fraturas vertebrais compressivas são comuns nos idosos, atingindo aproximadamente 1,5 milhão de novos casos na população americana. ${ }^{1}$ Cerca de $25 \%$ das mulheres pós-menopausa apresentam fraturas vertebrais durante o fim de sua vida. ${ }^{2} \mathrm{O}$ estudo EPOS registrou a incidência anual de casos, correlacionando os sexos, encontrando a relação de 10,7 acometidas a cada 1.000 mulheres e 5,7 acometidos a cada 1.000 homens. $^{3}$

As fraturas compressivas geralmente são provenientes de condições patológicas primárias como: hemangioma, mieloma múltiplo, metástases osteolíticas ou osteoporose primária e secundária. ${ }^{4}$ Os idosos, sem dúvida, são os mais acometidos por fraturas compressivas, sendo geralmente causadas por osteoporose moderada a grave.

A osteoporose caracteriza-se por redução da massa óssea e deterioração da microarquitetura do tecido ósseo, ocasionando fragilidade nos ossos e tornando-os mais suscetíveis a fraturas. ${ }^{5} \mathrm{O}$ aumento da idade proporciona alterações histológicas no osso, como a diminuição da estabilidade do colágeno trabecular e a alteração da orientação molecular dos proteoglicanos. ${ }^{6,7}$

As mulheres brancas na pós-menopausa são as que apresentam mais fraturas osteoporóticas. A partir dos 50 anos, $30 \%$ das mulheres e $13 \%$ dos homens podem apresentar fratura ao longo da vida. ${ }^{3,8}$ No Brasil, a incidência é similar, especialmente na população branca, sendo menos incidente na população negra. ${ }^{9}$

A manifestação mais comum da osteoporose é a fratura patológica. Forças mínimas, quando aplicadas no osso descontínuo e enfraquecido, são capazes de causar fraturas. ${ }^{10}$ Embora as fraturas por compressão aguda dos corpos vertebrais sejam dolorosas, a intensidade da dor em pacientes osteoporóticos é variável, sendo o risco de desenvolvimento de dor crônica diretamente relacionado ao número de fraturas vertebrais. ${ }^{11} \mathrm{O}$ colapso vertebral osteoporótico de vértebras adjacentes ainda pode cursar com instabilidade e/ou cifose, resultando em diminuição do apetite, má nutrição, diminuição da função pulmonar e alterações psicossociais. ${ }^{8,12}$

O declínio progressivo do estado de saúde contribui para o aumento da morbidade e mortalidade em pacientes com fraturas osteoporóticas em comparação com a população geral. ${ }^{4,5}$ As fraturas osteoporóticas aumentam também os custos médicos. Nos Estados Unidos, estima-se que são gastos 746 milhões de dólares por ano para o tratamento dos pacientes com fraturas osteoporóticas da coluna vertebral. ${ }^{6,7}$

\section{Etiologia}

O trauma, a infecção e as neoplasias podem causar fratura compressiva de vértebras, contudo a causa mais comum ainda é a osteoporose..$^{10,11,13} \mathrm{~A}$ redução da densidade mineral óssea, por alteração da microarquitetura óssea, e do conteúdo do colágeno, resultante das mudanças hormonais nas mulheres pós-menopausa, resulta no enfraquecimento ósseo e consequente aumento do risco de fraturas osteoporóticas. ${ }^{14} \mathrm{Um}$ levantamento epidemiológico nos Estados Unidos estimou que cerca de 44 milhões de pessoas têm osteoporose e cerca de 38 milhões têm redução da massa óssea. ${ }^{15}$

Alguns estudos sugerem que ter uma fratura compressiva aumenta as chances de uma nova fratura compressiva. Lindsay et al. ${ }^{16}$ identificaram que, independentemente da densidade do osso, uma ou mais fraturas aumenta cinco vezes o risco de o paciente apresentar nova fratura. Outro estudo relatou que uma fratura por compressão aumenta em $5 \%$ as chances de uma nova fratura, enquanto duas fraturas aumentam em $12 \%$ as chances de o paciente vir a ter outra fratura. ${ }^{17,18} \mathrm{~A}$ densidade mineral óssea também é fator de risco considerável e a redução de dois desvios standart acrescentam em quatro a seis vezes a chance de fraturas compressivas. ${ }^{17}$

\section{Fatores de risco}

Além da osteoporose como fator de risco, existe uma série de fatores modificáveis e não modificáveis. Entre os fatores modificáveis estão o etilismo, tabagismo, osteoporose, deficiência de estrogênio, menopausa precoce ou ooferectomia bilateral, amenorreia por mais de um ano, fraqueza, diminuição da acuidade visual, sedentarismo, deficiência de cálcio e vitamina $\mathrm{D}$ na dieta. ${ }^{19,20}$ Os fatores não modificáveis incluem a idade avançada, sexo feminino, raça branca, demência, suscetibilidade à queda, história de fraturas na idade adulta, história de fraturas em parentes de primeiro grau, tratamento anterior com esteroides ou anticonvulsivantes.

A obesidade, curiosamente, é protetora contra fraturas, uma vez que diminui o risco de perda óssea, pois, além da produção periférica de estrógeno, a alta tensão no osso induz a remodelação óssea. ${ }^{21} \mathrm{~A}$ hiperinsulinemia leva ao aumento dos níveis de IGF-1, o qual estimula a proliferação de osteoblastos. ${ }^{21}$

\section{Manifestações clínicas das fraturas vertebrais osteoporóticas}

O principal sintoma presente é a dor; já os déficits neurológicos tendem a ser pouco frequentes, pois, dificil- 
mente, a fratura envolve retropulsão de fragmentos ósseos para dentro do canal vertebral. As fraturas vertebrais em pacientes osteoporóticos geralmente acontecem em eventos triviais, como levantar objetos leves, tosse vigorosa ou espirrar ou até mesmo se virar na cama. Alguns pesquisadores acreditam na hipótese de que as fraturas vertebrais em pacientes osteoporóticos se dão por contração vigorosa da musculatura paraespinhal. ${ }^{22-24}$ Garfin et al. ${ }^{25}$ sugeriram que cerca de $30 \%$ das fraturas vertebrais em pacientes osteoporóticos graves acontecem quando o paciente está dormindo. Já os pacientes com osteoporose moderada podem apresentar fraturas vertebrais ao cair de uma cadeira, tropeçar ou ao levantar um objeto pesado. As chances de o paciente osteoporótico ter compressão medular aumenta com a gravidade do trauma, tal como um acidente de carro ou uma queda de grande altura. ${ }^{26}$

As fraturas de vértebras osteoporóticas têm início insidioso e podem se manifestar apenas com dor nas costas de leve intensidade. Ao longo do tempo, as múltiplas fraturas podem levar à perda progressiva da estatura, além de ocasionar contração contínua da musculatura paraespinhal, a fim de tentar manter a postura. Essa situação acaba fadigando a musculatura e causando aumento da dor. ${ }^{27}$

Os pacientes com múltiplas fraturas vertebrais apresentam perda progressiva da altura vertebral, desenvolvendo excessiva cifose torácica e lordose lombar. ${ }^{22}$ Nos casos mais graves a cifose é tão intensa que a pressão da cavidade torácica sobre a pelve resulta na redução da complacência pulmonar, abdome protuberante, saciedade precoce e perda de peso.
Constipação, obstrução intestinal, inatividade prolongada, trombose venosa profunda, fraqueza muscular progressiva, perda da independência, aprisionamento dos órgãos internos, distúrbios respiratórios, baixa autoestima e problemas sociais são complicações que podem advir de fraturas de vértebras osteoporóticas. ${ }^{21}$ Meunier et al. ${ }^{18}$ observaram que pacientes com fraturas de vértebras osteoporóticas têm risco de morte 15\% maior do que aqueles que não têm fratura vertebral.

Cerca de $60 \%$ a $75 \%$ das fraturas de vértebras osteoporóticas ocorrem na coluna toracolombar. O segmento mais acometido está entre T12 e L2, que é considerado a zona de transição entre uma estrutura mais rígida e outra mais móvel. Essa relação torna essa região mais propensa às fraturas compressivas, quando comparadas aos outros segmentos vertebrais. O quadro 1 resume as manifestações clínicas e as complicações das FVO.

\section{Classificação das fraturas vertebrais}

As fraturas osteoporóticas vertebrais podem ser classificadas em três categorias: cunha, bicôncava e esmagamento. As fraturas em cunha são as mais comuns, representando cerca de $50 \%$ de todas as fraturas vertebrais compressivas. ${ }^{28}$ Essas fraturas ocorrem mais na região torácica e são caracterizadas por compressão do segmento anterior do corpo vertebral. As fraturas

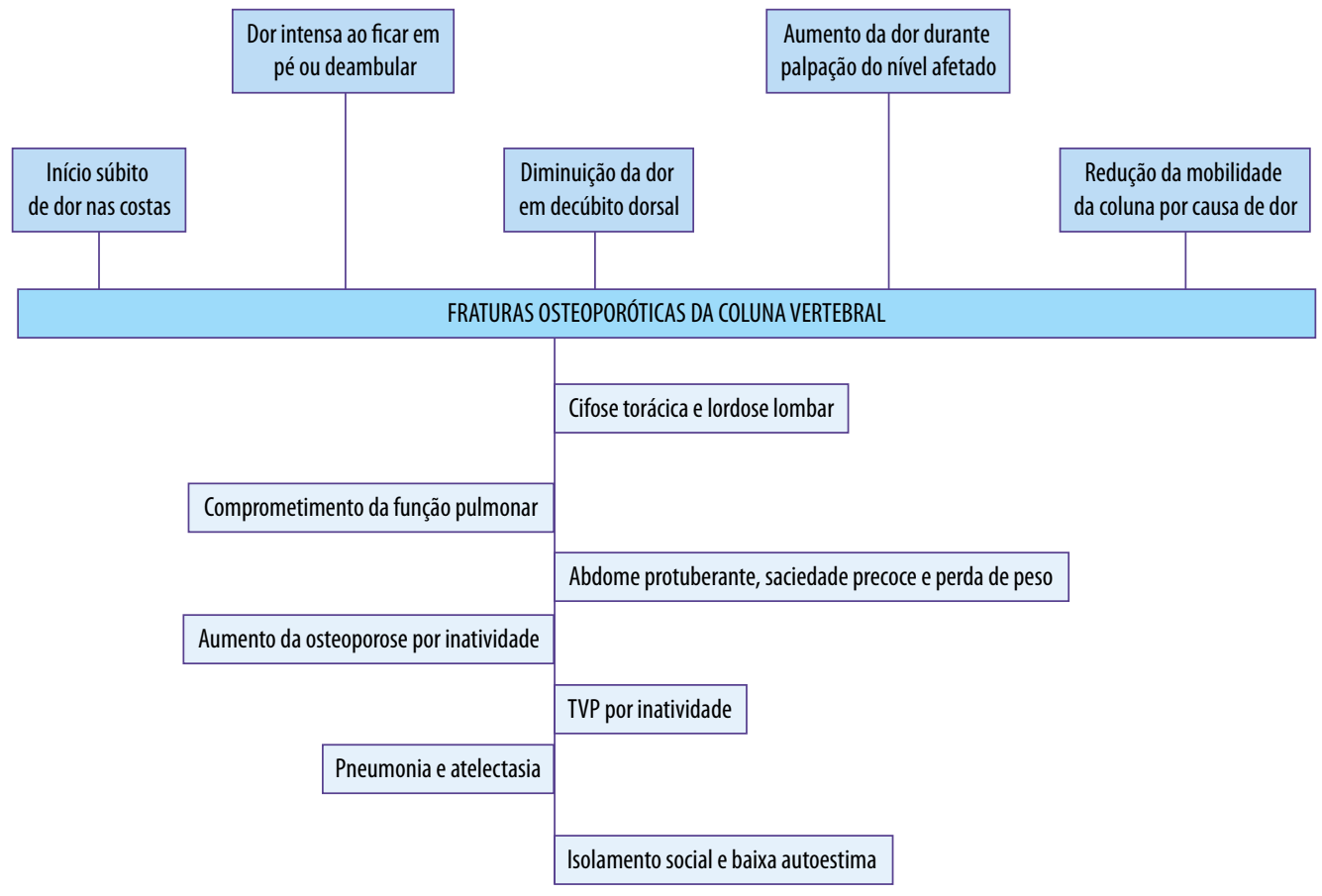

Quadro 1 - Sintomas e complicações decorrentes das fraturas osteoporóticas da coluna vertebral. 
bicôncavas representam cerca de $17 \%$ das fraturas vertebrais compressivas. Nestas fraturas somente a parte do meio do corpo vertebral é atingida, enquanto as paredes anterior e posterior permanecem intactas. As fraturas por esmagamento representam $13 \%$ de todas as fraturas compressivas, são caracterizadas por comprometer todo o corpo vertebral, incluindo margens anterior e posterior. As fraturas complexas representam os outros $20 \%$ das fraturas compressivas. ${ }^{28}$

\section{Diagnóstico da osteoporose}

O método mais confiável para se efetuar o diagnóstico de osteoporose nos pacientes com risco de desenvolver fraturas vertebrais é a medida da densidade mineral óssea. Atualmente, o padrão-ouro para medir a densidade mineral óssea é a DEXA (dual energy X-ray absorptiometry). ${ }^{29}$ Esse exame se tornou o de escolha, porque permite medir a massa central óssea com excelente especificidade. $\mathrm{O}$ método utilizado para a aferição da densidade é o T-score, que se refere ao pico de massa óssea em adultos jovens, sendo os desvios-padrão utilizados como modo de medição quantitativa da densidade mineral óssea. De acordo com a Organização Mundial de Saúde (OMS), T-score menor que -2,5 indica osteoporose, enquanto T-score de - 1 a $-2,5$ indica osteopenia ou redução da densidade óssea, ${ }^{29}$ e valores de T-score superiores a - 1 são normais.

\section{Exames de imagem para diagnóstico de fraturas vertebrais}

Existem várias modalidades de exames de imagem disponíveis que podem auxiliar no diagnóstico das fraturas vertebrais osteoporóticas. Inicialmente, podese utilizar a radiografia simples. Todo paciente com suspeita de fratura vertebral deve ter uma radiografia lombar total, pois tal exame simples evita que fraturas sejam negligenciadas por causa de outras doenças. ${ }^{26} \mathrm{~A}$ perda da altura vertebral, alterações do alinhamento anterior ou posterior, luxações e aumento da distância interespinhal $(>7 \mathrm{~mm})$ são indicadores de fraturas vertebrais. $^{2}$ Além de identificar as lesões já citadas, a radiografia em perfil permite identificar o grau de angulação cifótica proveniente da fratura; tal parâmetro auxilia no acompanhamento terapêutico dos pacientes.

A tomografia computadorizada (TC) também tem sua indicação especialmente nos casos em que a radio- grafia sugere lesão vertebral. A TC ajuda a identificar áreas de instabilidade e imagens de compressão em cunha e lesões ósseas ocultas. A TC ainda é ideal para fraturas complexas e para determinar o grau de compressão vertebral. ${ }^{30}$

As modalidades mais complexas como TC com mielografia e ressonância nuclear magnética (RNM) são reservadas para os casos em que há comprometimento neurológico. Nos casos em que as fraturas são decorrentes de doença infecciosa ou processos malignos, a RNM pode ser utilizada. Outra importante utilidade da RNM é para determinar a idade da FVO. Novas lesões são identificadas como aumento do sinal em T2, decorrente do aumento de líquido presente no corpo vertebral fraturado. Nos pacientes com contraindicações para a realização da RNM, pode ser realizada a TC com mielografia. $^{31}$

\section{Tratamento da osteoporose}

$\mathrm{O}$ tratamento ideal para as fraturas de vértebras osteoporóticas consiste na prevenção e no tratamento da osteoporose. As mulheres pós-menopausadas com osteoporose devem ser tratadas com $1.500 \mathrm{mg}$ de cálcio e 400 UI de vitamina $D$ diariamente. ${ }^{18,22}$

Nos homens com fraturas de vértebras, deve-se descartar hipogonadismo por meio da dosagem de testosterona. ${ }^{18,22} \mathrm{~A}$ osteomalácia deve ser suspeitada se os níveis de fosfatase alcalina estiverem elevados. $\mathrm{O}$ tabagismo deve ser desencorajado e aconselhado o uso do álcool de forma moderada. ${ }^{18}$ Exercícios físicos diários devem ser recomendados. ${ }^{22}$ Recentemente, surgiram como novas opções farmacológicas para o controle da osteoporose os bifosfonados, que reduzem o risco de fraturas. ${ }^{14,18}$ Ensaios clínicos randomizados mostraram que $\mathrm{o}$ alendronato reduz o risco de fratura vertebral em $50 \%$ das mulheres pós-menopausadas. ${ }^{32}$ Outros agentes que têm mostrado sucesso na prevenção de fraturas são o raloxifeno, paratormônio (PTH) e calcitonina. ${ }^{33}$

\section{Tratamento não cirúrgico}

A escolha do tratamento não cirúrgico ainda é a abordagem preferida para o tratamento das FVO. ${ }^{16,34}$ $\mathrm{O}$ tratamento conservador inclui períodos curtos de repouso, seguido pela mobilização gradual por órteses externas. ${ }^{33} \mathrm{Se}$ as $\mathrm{FVO}$ forem decorrentes do processo de compressão por flexão, a cinta de hiperextensão é utilizada. Esses processos são benéficos nos primeiros 
meses, para o controle da dor. Tais medidas são mais bem toleradas pelos pacientes mais jovens, ao contrário dos idosos, que apresentam aumento da dor com o uso das órteses. ${ }^{34}$ Dessa forma, os pacientes idosos acabam exigindo maior repouso, aumento da sua restrição ao leito, de forma a predispor a trombose venosa e embolia pulmonar. Podem resultar em úlceras de pressão, complicações pulmonares, infecção do trato urinário e sarcopenia progressiva. Além disso, alguns estudos têm mostrado que a restrição ao leito reduz em $0,25 \%$ a $1 \%$ a densidade mineral óssea por semana. ${ }^{24,35}$

Como forma de tentar reduzir a dor e promover o início da mobilização, deve-se prescrever corretamente o uso de analgésicos. Os opioides devem ser reservados para os pacientes que não controlam a dor com analgésicos comuns. As principais preocupações com o uso dos opioides se devem à dependência física e aos efeitos adversos como dismotilidade gastrointestinal e déficits cognitivos.

A fisioterapia e a reabilitação são fatores que aceleram a cicatrização. A radioterapia para o controle da dor pode ser utilizada em algumas causas de fraturas vertebrais não osteoporóticas como fraturas por mieloma múltiplo, metástase de próstata ou tumores ósseos radiossensíveis. ${ }^{36,37}$

Vescini e Grimaldi demonstraram os benefícios do uso do PTH no tratamento das FVO. O PTH, de forma intermitente, exerce ação anabólica mais rápida e maior que a ação catabólica tradicional. Dessa forma, o PTH reduz o risco relativo de fraturas osteoporóticas e aumenta a densidade mineral óssea, em especial no osso esponjoso. Outro efeito positivo do PTH consiste na cicatrização das fraturas, mostrada tanto pelo tempo reduzido no reparo das fraturas como pela melhoria em todos os parâmetros de formação do calo ósseo. Embora tais efeitos tenham sido comprovados em animais, alguns estudos em humanos já confirmaram em parte esses resultados. Nos pacientes idosos, acredita-se que o tratamento com PTH reduz o tempo de cicatrização, além de melhorar os resultados clínicos e reduzir a imobilização, diminuindo também as complicações decorrentes da imobilização. ${ }^{38}$

\section{Tratamento cirúrgico}

O manejo cirúrgico das fraturas osteoporóticas produz rápidas melhorias na qualidade de vida, uma vez que reduz as dores e melhora a função e a mobilidade dos pacientes. ${ }^{39}$

O tratamento cirúrgico é indicado para:

- dor não controlada pelo manejo não cirúrgico;

- déficits neurológicos;

- deformidade severa.
Contudo, os pacientes idosos possuem mais restrições para o tratamento operatório em decorrência de suas comorbidades. ${ }^{22,40}$ Os procedimentos mais populares para o controle da dor são a cifoplastia e a vertebroplastia percutânea. ${ }^{40,41}$ Outros métodos incluem o uso de fixadores espinhais para redução das fraturas (Alphatec Spine, Carlsbad, CA) e órteses internas. As formas mais invasivas, como a descompressão com colocação de parafusos, placas, gaiolas e hastes, também estão disponíveis. Contudo, esses procedimentos são ainda um desafio para o manejo de fraturas osteoporóticas.

A vertebroplastia percutânea é o método mais popular; consiste na injeção de polimetilmetacrilato (PMMA), provocando aumento do corpo vertebral e redução da dor. Alguns estudos mostraram que $75 \%$ a $100 \%$ dos pacientes submetidos a esse procedimento tiveram bom ou moderado controle da dor, aumentando a capacidade funcional pela estabilização da fratura e prevenção do colapso vertebral. ${ }^{42,43}$ As contraindicações para esse procedimento são infecções do corpo vertebral, coagulopatia, fraturas em retropulsão e alergia ao cimento de PMMA ou ao contraste. A principal complicação da injeção óssea de PMMA é o vazamento do cimento, que varia de $3 \%$ a $75 \%$. Esse vazamento pode ocasionar déficits neurológicos, tais como radiculopatias ou compressão medular. Além disso, há relatos de aumento da incidência de novas FVO dos segmentos adjacentes. ${ }^{23}$

Um estudo retrospectivo com 147 pacientes que evoluíram com novas fraturas de vértebras osteoporóticas (NFVO) tentou identificar os prováveis fatores de risco. Observou-se que $18,4 \%$ tiveram NFVO sintomáticas com tempo médio de 70 dias, $85 \%$ tiveram fraturas assintomáticas em um ano e $66,7 \%$ dos pacientes com NFVO tiveram fraturas de vértebras adjacentes. Os fatores de risco identificados foram a osteoporose e o vazamento discal de PMMA. ${ }^{44}$

Em 2009, Buchbinder et al. ${ }^{45}$ descobriram que a vertebroplastia não ofereceu benefício para o controle da dor de FVO. Nesse estudo controlado por placebo, os pesquisadores realizaram cirurgia fictícia, que consistiu em inserção percutânea da agulha e preparo do PMMA para liberar o odor para, dessa forma, simular uma operação real. ${ }^{45}$ Os pacientes de ambos o grupos apresentaram reduções significativas da dor e melhora da funcionalidade física e da qualidade de vida ${ }^{46}$ Outro estudo semelhante também mostrou que a vertebroplastia e um procedimento fictício tiveram resultados equivalentes. ${ }^{46}$

A cifoplastia é outra opção para o aumento do corpo vertebral. Consiste na colocação de um balão inflável nas fraturas vertebrais. ${ }^{27} \mathrm{O}$ balão é inflado e com um meio de contraste a posição e o enchimento do balão são confirmados. O balão cria uma cavidade que posteriormente pode ser preenchida por PMMA ou outros 
tipos de cimentos ósseos. Os riscos desse procedimento são semelhantes aos da vertebroplastia percutânea, porém apresentam menos índices de vazamento do cimento para dentro da medula. ${ }^{39} \mathrm{~A}$ cifoplastia reverte a deformidade espinhal, melhorando o pós-operatório de $50 \%$ a $70 \%$ e a cifose segmentar em $6^{\circ}$ a $10^{\circ} .^{26,47}$ Dessa forma, a cifoplastia tem a capacidade de reduzir as complicações pulmonares e gastrointestinais associadas às fraturas vertebrais. ${ }^{47}$ Se realizada dentro de três meses da ocorrência da fratura, a cifoplastia possui mais chances de restaurar a altura da vértebra fraturada. ${ }^{23,24}$ Cerca de $85 \%$ a $100 \%$ dos pacientes possuem bom ou moderado controle da dor. ${ }^{26}$ As contraindicações desse procedimento incluem as infecções do corpo vertebral, coagulopatias, retropulsão do fragmento ósseo, alergia às substâncias utilizadas no procedimento, incluindo o cimento e o contraste. ${ }^{25}$ As complicações em curto prazo desse procedimento foram relacionadas ao extravasamento do cimento e danos pelo calor e pressão sobre a medula espinhal e nervos adjacentes. ${ }^{39} \mathrm{~A}$ tabela 1 resume os estudos e conclusões referentes a vertebroplastia e cifoplastia.

\begin{tabular}{lcc}
\hline \multicolumn{2}{c}{$\begin{array}{c}\text { Tabela } 1 \text { - Risco de fraturas adjacentes } \\
\text { submetidos à cifoplastia e vertebroplastia }\end{array}$} \\
\hline Risco de fratura \% & Follow- up (meses) & Autor (ano) \\
\hline Sem tratamento & 6 & Kasperk et al. ${ }^{48}(2005)$ \\
12 & 12 & Lindsay et al. ${ }^{49}(2001)$ \\
19,2 & & \\
Vertebroplastia & 24 & Uppin et al. ${ }^{50}(2003)$ \\
8,3 & 48 & Grados et al..$^{51}(2000)$ \\
52 & & \\
Cifoplastia & 18 & Fribourg et al. ${ }^{52}(2004)$ \\
28 & 6 & Kasperk et al. ${ }^{48}(2005)$ \\
6 & &
\end{tabular}

Em 2009, foi desenvolvida uma nova técnica que busca diminuir as taxas de vazamento de cimento, a vesselplasty. $\mathrm{O}$ balão é inflado e deixado no paciente e preenchido pelo cimento, reduzindo, assim, o risco de vazamento. ${ }^{53}$

Novos estudos tentam demonstrar que o cimento de fosfato de cálcio (CFC) é mais seguro que PMMA. Um estudo com cadáveres demonstrou que o CFC aumenta a força vertebral, em 10 dias após o implante, quando comparado com outro grupo de vértebras intactas. ${ }^{54} \mathrm{O} C F C$ possui força de compressão máxima de $80 \mathrm{MPa}$, enquanto o PMMA possui força de compressão máxima de 99 $\mathrm{MPa}$; do ponto de vista radiológico, tem se demonstrado que o uso do PMMA tem mostrado melhores resultados que o CFC. ${ }^{55}$ Mesmo com os riscos semelhantes aos do PMMA os defensores dessa técnica acreditam que o CFC é mais biocompatível, biodegradável e mais osteo- construtor, sendo, portanto, mais seguro que o PMMA. Porém, existem controvérsias, em parte por causa da manipulação do CFC, que difere do PMMA; o custo, o desempenho e o longo tempo de endurecimento, além de ser mais difícil injetar o CFC sob alta pressão, são características que desestimulam o seu uso. ${ }^{56}$

Em seu estudo sobre o CFC, Nakano et al..$^{56}$ concluíram que, quando utilizado em pacientes cuidadosamente selecionados, usando-se a quantidade apropriada de pó/líquido injetado, impediu o colapso do corpo vertebral e pseudoartrose tardia, oferecendo resultados satisfatórios após longo período de follow-up. Contudo, as limitações do estudo consistem no fato de não haver um grupo controle. Recentemente, um estudo de coorte prospectivo não observou diferenças estatísticas significativas entre CFC e PMMA em cifoplastia para pacientes com fratura dolorosa de vértebras osteoporóticas, não havendo diferença significativa entre o VAS-score, Mobility-score ou height-restoration. ${ }^{57}$

Devido ao risco de fraturas adjacentes após a injeção do PMMA ou CFC, outra frente de estudos procura uma forma de reduzir os riscos de colapsos adjacentes. Sendo assim, Tseng et al. ${ }^{57}$ estudaram os agentes antirreabsorção como forma de prevenir as NFVO e perceberam que a maioria das novas fraturas ocorre dentro de dois a três meses e que os agentes antirreabsorção não protegem contra seu desenvolvimento. Contudo, em seu estudo, concluíram que o teriparatide aumentou em $21,7 \%$ a densidade mineral óssea, após 18 meses de tratamento, e a redução do risco de fraturas foi de $78,57 \%$. A terapia com teriparatide aumentou significativamente o JOA-score e reduziu o VAS-score. Acredita-se que o efeito terapêutico do teriparatide é melhor que o da vertebroplastia combinada com um tratamento antirreabsorção, tendo grande potencial para prevenção de NFVO após vertebroplastias.

\section{Considerações finais}

Percebe-se que ainda há controvérsias a serem discutidas e analisadas por estudos mais completos, para que seja definida qual a melhor opção terapêutica a ser adotada. Mesmo com novos estudos que procuram opções que reduzam os riscos inerentes aos procedimentos existentes, nada ainda demonstrou ser mais efetivo que a vertebroplastia e a cifoplastia com PMMA. Contudo, por haver riscos inerentes a esses procedimentos, eles devem ser indicados de forma correta. A associação de drogas que reduzem a chance de NFVO adjacentes parece ser a frente de estudos que mais levanta expectativas para o controle ideal da dor e retorno dos pacientes ao convívio e atividades diárias. 


\section{Referências}

1. Barr JD, Barr MS, Lemley TJ, McCann RM. Percutaneous vertebroplasty for pain relief and spinal stabilization. Spine (Phila Pa 1976). 2000;25(8):923-8.

2. Greenberg M. Handbook of neurosurgery. 6th ed. Nova York: Thieme Publishing Group; 2005.

3. European Prospective Osteoporosis Study (EPOS) Group; Felsenberg D, Silman AJ, Lunt M, Armbrecht G, Ismail AA, Finn JD, et al. Incidence of vertebral facture in Europe: results from the European Prospective Osteoporosis Study (EPOS). J Bone Miner Res. 2002;17(4):716-24.

4. Kado DM, Browner WS, Palermo L, Nevitt MC, Genant HK, Cummings SR. Vertebral fractures and mortality in older women: a prospective study. Study of Osteoporotic Fractures Research Group. Arch Intern Med. 1999;159(11):1215-20.

5. Huang MH, Barrett-Connor E, Greendale GA, Kado DM. Hyperkyphotic posture and risk of future osteoporotic fractures: the Rancho Bernardo study. J Bone Miner Res. 2006;21(3):419-23.

6. Melton LJ 3rd. Epidemiology of spinal osteoporosis. Spine (Phila Pa 1976). 1997;22(Suppl 24):2S-11S.

7. Lad SP, Patil CG, Lad EM, Boakye M. Trends in pathological vertebral fractures in the United States: 1993 to 2004. J Neurosurg Spine. 2007;7(3):305-10.

8. Kanis JA. Diagnosis of osteoporosis and assessment of fracture risk. Lancet. 2002;359(9321):1929-36.

9. Fourney DR, Schomer DF, Nader R, Chlan-Fourney J, Suki D, Ahrar K, et al. Percutaneous vertebroplasty and kyphoplasty for painful vertebral body fractures in cancer patients. J Neurosurg. 2003;98(Suppl 1):21-30.

10. Robinson Y, Heyde CE, Försth P, Olerud C. Kyphoplasty in osteoporotic vertebral compression fractures - guidelines and technical considerations. J Orthop Surg Res. 2011;6:43.

11. Nevitt MC, Ettinger B, Black DM, Stone K, Jamal SA, Ensrud $\mathrm{K}$, et al. The association of radiographically detected vertebral fractures with back pain and function: a prospective study. Ann Intern Med. 1998;128(10):793800.

12. Cummings SR, Melton LJ. Epidemiology and outcomes of osteoporotic fractures. Lancet. 2002;359(9319):1761-7.

13. Gertzbein SD, Khoury D, Bullington A, St John TA, Larson Al. Thoracic and lumbar fractures associated with skiing and snowboarding injuries according to the $\mathrm{AO}$ Comprehensive Classification. Am J Sports Med. 2012;40(8):1750-4.

14. Resch A, Schneider B, Bernecker P, Battmann A, Wergedal $J$, Willvonseder $R$, et al. Risk of vertebral fractures in men: relationship to mineral density of the vertebral body. AJR Am J Roentgenol. 1995;164(6):1447-50.

15. Qaseem A, Snow V, Shekelle P, Hopkins R Jr, Forciea MA, Owens DK, et al. Pharmacologic treatment of low bone density or osteoporosis to prevent fractures: a clinical practice guideline from the American College of Physicians. Ann Intern Med. 2008;149(6):404-15.

16. Lindsay R, Silverman SL, Cooper C, Hanley DA, Barton I, Broy SB, et al. Risk of new vertebral fracture in the year following a fracture. JAMA. 2001;285(3):320-3.

17. Marshall $\mathrm{D}$, Johnell $\mathrm{O}$, Wedel H. Meta-analysis of how well measures of bone mineral density predict occurrence of osteoporotic fractures. BMJ. 1996;312(7041):1254-9.

18. Meunier PJ, Delmas PD, Eastell R, McClung MR, Papapoulos S, Rizzoli R, et al. Diagnosis and management of osteoporosis in postmenopausal women: clinical guidelines. International Committee for Osteoporosis Clinical Guidelines. Clin Ther. 1999;21(6):1025-44.
19. Garfin SR, Reilley MA. Minimally invasive treatment of osteoporotic vertebral body compression fractures. Spine J. 2002;2(1):76-80

20. Melton LJ 3rd. Epidemiology of osteoporosis: predicting who is at risk. Ann N Y Acad Sci. 1990;592:295-306.

21. Theodorou DJ, Theodorou SJ, Duncan TD, Garfin SR, Wong $\mathrm{WH}$. Percutaneous balloon kyphoplasty for the correction of spinal deformity in painful vertebral body compression fractures. Clin Imaging. 2002;26(1):1-5.

22. Kim DH, Vaccaro AR. Osteoporotic compression fractures of the spine; current options and considerations for treatment. Spine J. 2006;6(5):479-87.

23. Shen MS, Kim YH. Vertebroplasty and kyphoplasty: treatment techniques for managing osteoporotic vertebral compression fractures. Bull NYU Hosp Jt Dis. 2006;64(34):106-13.

24. Leblanc AD, Schneider VS, Evans HJ, Engelbretson DA, Krebs JM. Bone mineral loss and recovery after 17 weeks of bed rest. J Bone Miner Res. 1990;5(8):843-50.

25. Garfin SR, Yuan HA, Reiley MA. New technologies in spine: kyphoplasty and vertebroplasty for the treatment of painful osteoporotic compression fractures. Spine (Phila Pa 1976). 2001;26(14):1511-5.

26. Lieberman IH, Dudeney S, Reinhardt MK, Bell G. Initial outcome and efficacy of "kyphoplasty" in the treatment of painful osteoporotic vertebral compression fractures. Spine (Phila Pa 1976). 2001;26(14):1631-8.

27. Wu SS, Lachmann E, Nagler W. Current medical, rehabilitation, and surgical management of vertebral compression fractures. J Womens Health (Larchmt). 2003;12(1):17-26.

28. Black DM, Arden NK, Palermo L, Pearson J, Cummings SR. Prevalent vertebral deformities predict hip fractures and new vertebral deformities but not wrist fractures. Study of Osteoporotic Fractures Research Group. J Bone Miner Res. 1999;14(5):821-8.

29. Slemenda CW, Hui SL, Longcope C, Wellman H, Johnston CC Jr. Predictors of bone mass in perimenopausal women. A prospective study of clinical data using photon absorptiometry. Ann Intern Med. 1990;112(2):96-101.

30. Epstein O, Ludwig S, Gelb D, Poelstra K, O'Brien J. Comparison of computed tomography and plain radiography in assessing traumatic spinal deformity. $J$ Spinal Disord Tech. 2009;22(3):197-201.

31. Alexandru D, So W. Evaluation and management of vertebral compression fractures. Perm J. 2012;16(4):46-51.

32. Black DM, Cummings SR, Karpf DB, Cauley JA, Thompson DE, Nevitt MC, et al. Randomised trial of effect of alendronate on risk of fracture in women with existing vertebral fractures. Fracture Intervention Trial Research Group. Lancet. 1996;348(9041):1535-41.

33. Gardner MJ, Demetrakopoulos D, Shindle MK, Griffith $\mathrm{MH}$, Lane JM. Osteoporosis and skeletal fractures. HSS J. 2006;2(1):62-9.

34. Truumees E, Hilibrand A, Vaccaro AR. Percutaneous vertebral augmentation. Spine J. 2004;4(2):218-29.

35. Krølner B, Toft B. Vertebral bone loss: an unheeded side effect of therapeutic bed rest. Clin Sci (Lond). 1983;64(5):537-40.

36. Sundaresan N, Krol G, Steinberger AA, Moore F. Management of tumors of the thoracolumbar spine. Neurosurg Clin N Am. 1997;8(4):541-53.

37. Sundaresan N, Schmidek HH, Schiller AL, Rosenthal $\mathrm{DI}$, editors. Tumors of the spine: diagnosis and clinical management. Philadelphia: Saunders; 1990. 
38. Vescini F, Grimaldi F. PTH 1-84: bone rebuilding as a target for the therapy of severe osteoporosis. Clin Cases Miner Bone Metab. 2012;9(1):31-6.

39. Garfin SR, Buckley RA, Ledlie J, Balloon Kyphoplasty Outcomes Group. Balloon kyphoplasty for symptomatic vertebral body compression fractures results in rapid, significant, and sustained improvements in back pain, function, and quality of life for elderly patients. Spine (Phila Pa 1976). 2006;31(19):2213-20.

40. Bostrom MP, Lane JM. Future directions. Augmentation of osteoporotic vertebral bodies. Spine (Phila Pa 1976). 1997;22(Suppl 24):38S-42S.

41. Vaccaro AR, Kim DH, Brodke DS, Harris M, Chapman JR, Schildhauer T, et al. Diagnosis and management of thoracolumbar spine fractures. Instr Course Lect. 2004;53:359-73.

42. McKiernan F, Faciszewski T, Jensen R. Quality of life following vertebroplasty. J Bone Joint Surg Am. 2004;86A(12):2600-6.

43. Lo YP, Chen WJ, Chen LH, Lai PL. New vertebral fracture after vertebroplasty. J Trauma. 2008;65(6):1439-45.

44. Rho YJ, Choe WJ, Chun YI. Risk factors predicting the new symptomatic vertebral compression fractures after percutaneous vertebroplasty or kyphoplasty. Eur Spine J. 2012;21(5):905-11.

45. Buchbinder R, Osborne RH, Ebeling PR, Wark JD, Mitchell $\mathrm{P}$, Wriedt $\mathrm{C}$, et al. A randomized trial of vertebroplasty for painful osteoporotic vertebral fractures. N Engl J Med. 2009;361(6):557-68.

46. Kallmes DF, Comstock BA, Heagerty PJ, Turner JA, Wilson DJ, Diamond TH, et al. A randomized trial of vertebroplasty for osteoporotic spinal fractures. N Engl J Med. 2009;361(6):569-79.

47. Gaitanis IN, Hadjipavlou AG, Katonis PG, Tzermiadianos MN, Pasku DS, Patwardhan AG. Balloon kyphoplasty for the treatment of pathological vertebral compressive fractures. Eur Spine J. 2005;14(3):250-60.

48. Kasperk C, Hillmeier J, Nöldge G, Grafe IA, Dafonseca K, Raupp D, et al. Treatment of painful vertebral fractures by kyphoplasty in patients with primary osteoporosis: a prospective nonrandomized controlled study. J Bone Miner Res. 2005;20(4):604-12.
49. Lindsay R, Silverman SL, Cooper C, Hanley DA, Barton I, Broy SB, et al. Risk of new vertebral fracture in the year following a fracture. JAMA. 2001;285(3):320-3.

50. Uppin AA, Hirsch JA, Centenera LV, Pfiefer BA, Pazianos AG, Choi IS. Occurrence of new vertebral body fracture after percutaneous vertebroplasty in patients with osteoporosis. Radiology. 2003;226(1):119-24.

51. Grados F, Depriester C, Cayrolle G, Hardy N, Deramond $\mathrm{H}$, Fardellone P. Long-term observations of vertebral osteoporotic fractures treated by percutaneous vertebroplasty. Rheumatology (Oxford). 2000;39(12):1410-4.

52. Fribourg D, Tang C, Sra P, Delamarter R, Bae H. Incidence of subsequent vertebral fracture after kyphoplasty. Spine (Phila Pa 1976). 2004;29(20):2270-6.

53. Flors L, Lonjedo E, Leiva-Salinas C, Martí-Bonmatí L, Martínez-Rodrigo JJ, López-Pérez E, et al. Vesselplasty: a new technical approach to treat symptomatic vertebral compression fractures. AJR Am J Roentgenol. 2009;193(1):218-26.

54. Ikeuchi M, Yamamoto H, Shibata T, Otani M. Mechanical augmentation of the vertebral body by calcium phosphate cement injection. J Orthop Sci. 2001;6(1):39-45.

55. Kindt-Larsen T, Smith DB, Jensen JS. Innovations in acrylic bone cement and application equipment. J Appl Biomater. 1995;6(1):75-83.

56. Nakano M, Hirano N, Zukawa M, Suzuki K, Hirose J, Kimura $\mathrm{T}$, et al. Vertebroplasty using calcium phosphate cement for osteoporotic vertebral fractures: study of outcomes at a minimum follow-up of two years. Asian Spine J. 2012;6(1):34-42.

57. Tseng YY, Su CH, Lui TN, Yeh YS, Yeh SH. Prospective comparison of the therapeutic effect of teriparatide with that of combined vertebroplasty with antiresorptive agents for the treatment of new-onset adjacent vertebral compression fracture after percutaneous vertebroplasty. Osteoporos Int. 2012;23(5):1613-22.

\section{Endereço para correspondência}

João Welberthon Matos Queiroz

Rua Padre Cícero, 715, Centro

63010-020 - Juazeiro do Norte, CE, Brasil

Telefone: (88) 8829-6961

E-mail: joaowelberthon@hotmail.com 